\title{
THREE DIMENSIONAL COMPLEX PERMITTIVITY RECONSTRUCTION BY MEANS OF NEWTON-TYPE MICROWAVE IMAGING
}

\author{
J. De Zaeytijd ${ }^{1}$, A. Franchois ${ }^{2}$, C. Eyraud ${ }^{3}$, and J-M. Geffrin ${ }^{3}$ \\ ${ }^{1}$ INTEC, Ghent University, Sint-Pietersnieuwstraat 41, 9000 Ghent, Belgium, Email: jurgen.dezaeytijd@intec.UGent.be \\ ${ }^{2}$ INTEC-IMEC, Ghent University, Sint-Pietersnieuwstraat 41, 9000 Ghent, Belgium, Email: \\ ann.franchois@intec.ugent.be \\ ${ }^{3}$ Institut Fresnel, UMR CNRSTIC 6133, Université Paul Cézanne Aix-Marseille III, Campus de Saint-Jérôme, case 162, \\ 13397 Marseille Cedex 20,France,Email: Christelle.Eyraud@fresnel.fr and Jean-Michel.Geffrin@fresnel.fr
}

\begin{abstract}
This paper treats the full-wave inverse electromagnetic scattering problem of complex permittivity reconstruction in three dimensions. Two Newton-type methods are used to solve this non-linear and ill-posed problem by minimizing a new type of regularized cost function. The Gauss-Newton method and the Broyden-FletcherGoldfarb-Shanno quasi-Newton algorithm are compared and results are presented, both from simulated and measured data.
\end{abstract}

Key words: microwave imaging, complex permittivity, inverse problems, nonlinear optimization, gauss-Newton optimization, quasi-Newton optimization, regularization.

\section{INTRODUCTION}

Most of the work done so far in the field of quantitative electromagnetic imaging has been concerned with $2 \mathrm{D}$ problems. As valuable as these explorations in two dimensions are to learn about the nature of inverse electromagnetic problems, sooner or later the step towards realistic full-vectorial 3D inversion must be taken. Moreover, indications arose recently that $3 \mathrm{D}$ measurements will become available in the near future [1]. Therefore in this paper a $3 \mathrm{D}$ complex permittivity reconstruction algorithm is presented. It uses Newton-type optimization algorithms to tackle the non-linear and ill-posed inverse problem, as is the reconstruction of inhomogeneous dielectric objects from scattering experiments. Newtontype algorithms were already used on 2D problems [2], [3] and are known to have valuable convergence properties. Since they involve matrix operations, the dimension of the optimization problem must be limited and therefore only the permittivity, which has to be reconstructed, is parameterized. Auxiliary variables, such as the field inside the reconstruction domain must be eliminated. To do so, several direct scattering problems must be solved. This can be a computationally demanding task in the 3D full-vectorial case, so attention is paid to efficient forward solvers. To alleviate the ill-posedness of the problem a regularized cost function is optimized which gives good reconstruction results. Two types of Newton-like methods are used on a generic example and compared: the Gauss-Newton method and the BroydenFletcher-Goldfarb-Shanno (BFGS) quasi-Newton algorithm. Finally a reconstruction from measured data is presented.

\section{FORMULATION OF THE INVERSE PROB- LEM}

Consider a 3D inhomogeneous lossy dielectric object situated in an infinite homogeneous and isotropic background (Fig. 1). For a fixed frequency $f=\omega / 2 \pi$ and from a number of source points $\boldsymbol{r}_{i_{s}}^{S}, i_{s}=1 \ldots N^{S}$, the object is illuminated by electric dipoles oriented along the directions $\hat{\boldsymbol{u}}_{i_{d}, i_{s}}^{D}, i_{d}=1 \ldots N^{D} \leq 3$. For every such excitation, the scattered field is measured in the set of receiver points $\boldsymbol{r}_{i_{r}}^{R}, i_{r}=1 \ldots N^{R}$ and along the directions $\hat{\boldsymbol{u}}_{i_{c}, i_{r}}^{C}, i_{c}=1 \ldots N^{C} \leq 3$. The objective is to reconstruct the complex permittivity distribution $\epsilon(\boldsymbol{r})=\epsilon^{\prime}(\boldsymbol{r})+j \epsilon^{\prime \prime}(\boldsymbol{r})$ within the volume $\mathcal{D}$ that encloses the object, i.e. $\epsilon(\boldsymbol{r})=\epsilon_{b}$, the background permittivity, whenever $\boldsymbol{r} \notin \mathcal{D}$.

It is well known that this inverse scattering problem is ill-posed, i.e. the existence, uniqueness and stability of the solution are not simultaneously guaranteed. Nonexistence, which is caused by noise on the measurements and the modeling error, is coped with by redefining the solution as the minimizer of a cost functional. Usually a least squares cost functional is applied:

$$
F(\epsilon)=\frac{1}{F_{0}}\left(\bar{E}^{s}(\epsilon)-\bar{E}^{m}\right)^{H}\left(\bar{E}^{s}(\epsilon)-\bar{E}^{m}\right),
$$

where $\bar{E}^{m}$ is a vector that contains the data and $\bar{E}^{s}(\epsilon)$ contains the calculated values of the field, scattered by a permittivity distribution $\epsilon(\boldsymbol{r}) .(\cdot)^{H}$ denotes the complex 


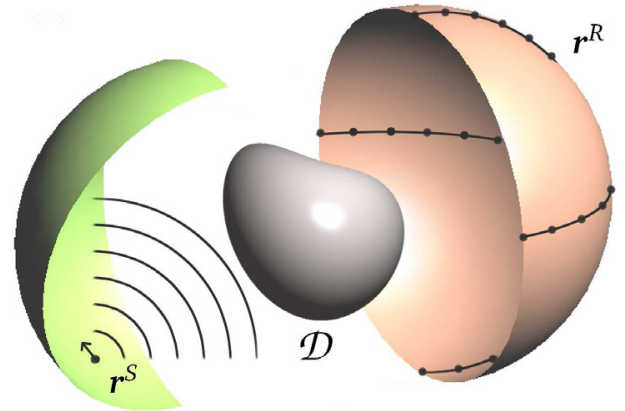

Figure 1. The 3D geometry for the inverse problem.

conjugate. Nonuniqueness can be solved theoretically by using "complete data", i.e. the fields are known in every point of a sphere around $\mathcal{D}$ and this for excitations in every point of such a surface [4]. Naturally in real applications such complete data is often not available and the optimization algorithm can also be trapped in local minima of the cost functional. Finally the solution of the inverse scattering problem as the minimizer of Eq. 1 is known to be very sensitive to noise on the data. Because of the above, a regularization of the problem is necessary and this will be discussed in more detail in section 6.1 .

\section{CONTRAST SOURCE FORMULATION}

The contrast source formulation is used to describe the scattering from the inhomogeneous object when it is illuminated by an incident field $\boldsymbol{e}^{i}(\boldsymbol{r})$ (the time dependency $e^{j \omega t}$ is omitted). Once the electric field $\boldsymbol{e}(\boldsymbol{r})$ in the object is known, the scattered field $e^{s}(r)$ is calculated as the field, generated by the so-called contrast currents:

$$
\begin{gathered}
\boldsymbol{j}^{s}(\boldsymbol{r})=j \omega\left[\epsilon(\boldsymbol{r})-\epsilon_{b}\right] \boldsymbol{e}(\boldsymbol{r})=j \omega \chi(\boldsymbol{r}) \boldsymbol{e}(\boldsymbol{r}), \\
\boldsymbol{e}^{s}(\boldsymbol{r})=-j \omega \mu_{b}\left[\mathbf{I}+\frac{1}{k_{b}} \nabla \nabla\right] \cdot \int_{\mathcal{D}} G_{b}\left(\boldsymbol{r}-\boldsymbol{r}^{\prime}\right) \boldsymbol{j}^{s}\left(\boldsymbol{r}^{\prime}\right) \mathrm{d} \boldsymbol{r}^{\prime},
\end{gathered}
$$

where $k_{b}$ is the background wavenumber and $\mu_{b}$ the background permeability. $G_{b}\left(\boldsymbol{r}-\boldsymbol{r}^{\prime}\right)$ is the Green function of the infinite background medium:

$$
G_{b}\left(\boldsymbol{r}-\boldsymbol{r}^{\prime}\right)=\frac{e^{-j k_{b}\left\|\boldsymbol{r}-\boldsymbol{r}^{\prime}\right\|}}{4 \pi\left\|\boldsymbol{r}-\boldsymbol{r}^{\prime}\right\|} .
$$

To know the total field $\boldsymbol{e}(\boldsymbol{r})$ in $\mathcal{D}$ and thus the contrast currents, the following equation is solved

$$
\boldsymbol{e}(\boldsymbol{r})=e^{i}(\boldsymbol{r})+\boldsymbol{e}^{s}(\boldsymbol{r}), \forall \boldsymbol{r} \in \mathcal{D}
$$

which is referred to as the domain equation. Eq. 3 is used to calculate the scattered field in the receiver points and is called the observation equation.

\section{PARAMETERIZATION}

In order to obtain a finite number of optimization variables, the permittivity profile $\epsilon(\boldsymbol{r})$ must be parameterized. First the domain $\mathcal{D}$ is discretized using a uniform cuboidal grid of $A \times B \times C$ cells. Then the contrast $\chi(\boldsymbol{r})$ is approximated by a piecewise constant function that has one value in each cuboidal cell:

$$
\chi(\boldsymbol{r})=\sum_{a=0}^{A-1} \sum_{b=0}^{B-1} \sum_{c=0}^{C-1}\left[\epsilon_{a, b, c}-\epsilon_{b}\right] \Psi_{a, b, c}(\boldsymbol{r}),
$$

where $\Psi_{a, b, c}(\boldsymbol{r})=1$ in cell $(a, b, c)$ and zero elsewhere. As for the fields in the domain $\mathcal{D}$, the electric flux density $\boldsymbol{d}(\boldsymbol{r})=\epsilon(\boldsymbol{r}) \boldsymbol{e}(\boldsymbol{r})$ rather than $\boldsymbol{e}(\boldsymbol{r})$ is expanded in 3D rooftop functions [5] on the same grid or on a finer grid.

\section{FORWARD PROBLEM}

In every iteration of the optimization process a multiview forward scattering problem has to be solved: for every excitation along $\hat{\boldsymbol{u}}_{i_{d}, i_{s}}^{D}$ in $\boldsymbol{r}_{i_{s}}^{S}$ the field $\boldsymbol{e}_{i_{d}, i_{s}}(\boldsymbol{r})$ in $\mathcal{D}$ has to be known to calculate $\boldsymbol{e}_{i_{d}, i_{s}}^{s}\left(\boldsymbol{r}_{i_{r}}^{R}\right) \cdot \hat{\boldsymbol{u}}_{i_{c}, i_{r}}^{C}$. Also the derivatives of these fields with respect to the optimization parameters $\epsilon_{a, b, c}$ are needed for the optimization. Analytical expressions for the derivatives can be obtained using reciprocity and this results in

$$
\begin{aligned}
& \frac{\partial \boldsymbol{e}_{i_{d}, i_{s}}^{s}\left(\boldsymbol{r}_{i_{r}}^{R}\right)}{\partial \epsilon_{a, b, c}} \cdot \hat{\boldsymbol{u}}_{i_{c}, i_{r}}^{C}= \\
& j \omega \int_{\mathcal{D}} \Psi_{a, b, c}\left(\boldsymbol{r}^{\prime}\right)\left(\boldsymbol{e}_{i_{d}, i_{s}}\left(\boldsymbol{r}^{\prime}\right) \cdot \boldsymbol{e}_{i_{c}, i_{r}}\left(\boldsymbol{r}^{\prime}\right)\right) \mathrm{d} \boldsymbol{r}^{\prime}
\end{aligned}
$$

where $\boldsymbol{e}_{i_{c}, i_{r}}(\boldsymbol{r})$ is the field in $\mathcal{D}$ due to a dipole excitation along $\hat{\boldsymbol{u}}_{i_{c}, i_{r}}^{C}$ in $\boldsymbol{r}_{i_{r}}^{R}$. This means that for every antenna, wether source or receiver, a forward problem must be solved. Since the solution of a full-vectorial 3D scattering problem is a computationally demanding task, two accelerating techniques are used. The FFT method [5] uses the convolution structure of Eq. 3 to accelarate the calculation of the scattered electric field in $\mathcal{D}$. In conjunction with an iterative solver this allows for rapid solution of Eq. 5. The Marching on in Angle method is an application of an extrapolation procedure described in [6]. The idea is to find a better initial estimate for the iterative solution of a linear system based on a number of previous solutions.

\section{THE OPTIMIZATION ALGORITHM}

\subsection{Regularization}

As mentioned in section 2 a regularization of the inverse problem is necessary. The parameterization of $\epsilon(\boldsymbol{r})$ itself already alleviates the ill-posedness of the problem 
by reducing the degrees of freedom. On top of this we propose an alternative cost functional $F_{R}(\epsilon)=F(\epsilon)(1+$ $\alpha \sigma(\epsilon))$. The functional $\sigma(\epsilon)$ is always positive and penalizes strong local variations in the permittivity distribution which can otherwise be induced by noisy data. In discretized form it is given by

$$
\begin{aligned}
\sigma(\epsilon)= & \sum_{a=0}^{A} \sum_{b=0}^{B-1} \sum_{c=0}^{C-1}\left|\epsilon_{a, b, c}-\epsilon_{a-1, b, c}\right|^{2} \\
& +\sum_{a=0}^{A-1} \sum_{b=0}^{B} \sum_{c=0}^{C-1}\left|\epsilon_{a, b, c}-\epsilon_{a, b-1, c}\right|^{2} \\
& +\sum_{a=0}^{A-1} \sum_{b=0}^{B-1} \sum_{c=0}^{C}\left|\epsilon_{a, b, c}-\epsilon_{a, b, c-1}\right|^{2}
\end{aligned}
$$

where $\epsilon_{a, b, c}=1$ if $a=-1, a=A, b=-1, b=B$, $c=-1$ or $c=C$. $\alpha$ is a positive real parameter that is chosen based on very general a priori knowledge, such as the maximal size of the object (already used to choose a domain $\mathcal{D}$ ) and an upper limit for the permittivity (already used to choose a mesh size for the forward problem).

\subsection{Newton Type Optimization Methods}

Let $\bar{\epsilon}$ and $\bar{\epsilon}^{*}$ be the vectors containing the coefficients $\epsilon_{a, b, c}$ from Eq. 6 and their complex conjugate respectively. For a real-valued function $F\left(\bar{\epsilon}, \bar{\epsilon}^{*}\right)$, the gradient vector $\bar{g}\left(\bar{\epsilon}, \bar{\epsilon}^{*}\right)$ and the hessian matrix $\overline{\overline{\boldsymbol{H}}}\left(\bar{\epsilon}, \bar{\epsilon}^{*}\right)$ are defined:

$$
\bar{g}\left(\bar{\epsilon}, \bar{\epsilon}^{*}\right)=\left[\begin{array}{c}
\frac{\partial F}{\partial \epsilon} \\
\frac{\partial F}{\partial \epsilon^{*}}
\end{array}\right], \quad \overline{\overline{\boldsymbol{H}}}\left(\bar{\epsilon}, \bar{\epsilon}^{*}\right)=\left[\begin{array}{cc}
\frac{\partial^{2} F}{\partial \epsilon \partial \epsilon} & \frac{\partial^{2} F}{\partial \epsilon \partial \epsilon^{*}} \\
\frac{\partial^{2} F}{\partial \epsilon^{*} \partial \epsilon} & \frac{\partial^{2} F}{\partial \epsilon^{*} \partial \epsilon^{*}}
\end{array}\right],
$$

In iteration $k$ of Newton's method the function $F\left(\bar{\epsilon}, \bar{\epsilon}^{*}\right)$ is approximated by a quadratic function, the minimum of which serves as next iterate:

$$
\left[\begin{array}{c}
\bar{\epsilon}_{k+1} \\
\bar{\epsilon}_{k+1}^{*}
\end{array}\right]=\left[\begin{array}{c}
\bar{\epsilon}_{k} \\
\bar{\epsilon}_{k}^{*}
\end{array}\right]+\left[\begin{array}{c}
\Delta \bar{\epsilon}_{k} \\
\Delta \bar{\epsilon}_{k}^{*}
\end{array}\right]=\left[\begin{array}{c}
\bar{\epsilon}_{k} \\
\bar{\epsilon}_{k}^{*}
\end{array}\right]-(\overline{\overline{\boldsymbol{H}}})_{k}^{-1} \bar{g}_{k}
$$

where the subscript $k$ indicates evaluation in $\left(\bar{\epsilon}_{k}, \bar{\epsilon}_{k}^{*}\right)$. Drawbacks of the method are the fact that convergence may not occur when far from the solution, $F_{k}=$ $F\left(\bar{\epsilon}_{k}, \bar{\epsilon}_{k}^{*}\right)$ may not even decrease, $\overline{\overline{\boldsymbol{H}}}_{k}$ may not be positive definite, which means that the quadratic approximation does not have a minimizer and second order derivatives of the scattered fields are needed, which cannot be easily and economically calculated.

The explicit expressions for $\bar{g}$ and $\overline{\overline{\boldsymbol{H}}}$ in case of the least squares cost function of Eq. 1 are:

$$
\begin{aligned}
\bar{g}\left(\bar{\epsilon}, \bar{\epsilon}^{*}\right) & =\frac{1}{F_{0}}\left[\begin{array}{l}
\overline{\overline{\boldsymbol{J}}}^{T}\left[\bar{E}^{s}(\bar{\epsilon})-\bar{E}^{m}\right]^{*} \\
\overline{\overline{\boldsymbol{J}}}^{H}\left[\bar{E}^{s}(\bar{\epsilon})-\bar{E}^{m}\right]
\end{array}\right] \\
\overline{\overline{\boldsymbol{H}}}\left(\bar{\epsilon}, \bar{\epsilon}^{*}\right) & =\frac{1}{F_{0}}\left[\begin{array}{cc}
\overline{\overline{\boldsymbol{B}}} & \overline{\overline{\boldsymbol{J}}}^{T} \overline{\overline{\boldsymbol{J}}}^{*} \\
\overline{\overline{\boldsymbol{J}}}^{H} \overline{\overline{\boldsymbol{J}}} & \overline{\overline{\boldsymbol{B}}}^{*}
\end{array}\right]
\end{aligned}
$$

with

$$
J_{i j}=\frac{\partial E_{i}^{s}}{\partial \epsilon_{j}} \quad \text { and } \quad B_{i j}=\frac{\partial^{2} \bar{E}^{s}}{\partial \epsilon_{i} \partial \epsilon_{j}}\left[\bar{E}^{s}(\bar{\epsilon})-\bar{E}^{m}\right]^{*} \text {. }
$$

$(\cdot)^{T}$ stands for transpose. When the submatrices $\overline{\bar{B}}$ in the Hessian matrix are neglected because of the appearance of $\left[\bar{E}^{s}(\bar{\epsilon})-\bar{E}^{m}\right]^{*}$, which should become small, one arrives at the Gauss Newton method. In iteration $k$, the updates are then given by (the index $k$ is omitted)

$$
\Delta \bar{\epsilon}=-\left(\overline{\overline{\boldsymbol{J}}}^{H} \overline{\overline{\boldsymbol{J}}}\right)^{-1} \overline{\overline{\boldsymbol{J}}}^{H}\left[\bar{E}^{s}(\bar{\epsilon})-\bar{E}^{m}\right] .
$$

Applying the same approximation for the regularized cost function $F_{R}\left(\bar{\epsilon}, \bar{\epsilon}^{*}\right)=F\left(\bar{\epsilon}, \bar{\epsilon}^{*}\right)\left(1+\alpha \sigma\left(\bar{\epsilon}, \bar{\epsilon}^{*}\right)\right)$, one arrives at

$$
\begin{aligned}
& \Delta \bar{\epsilon}=-\left(\overline{\overline{\boldsymbol{J}}}^{H} \overline{\overline{\boldsymbol{J}}}(1+\alpha \sigma)+\alpha F \overline{\overline{\boldsymbol{\Sigma}}}\right)^{-1} \times \\
& \left(\overline{\overline{\boldsymbol{J}}}^{H}\left[\bar{E}^{s}(\bar{\epsilon})-\bar{E}^{m}\right]+\alpha \bar{\Omega}\right),
\end{aligned}
$$

with

$$
\Sigma_{i j}=\frac{\partial^{2} \sigma}{\partial \epsilon_{i} \partial \epsilon_{j}^{*}}, \quad \text { and } \quad \Omega_{i}=\frac{\partial \sigma}{\partial \epsilon_{i}} .
$$

Note that no second order derivatives of the scattered fields are needed. Also, the reduced Hessian matrices in Eq. 14 and Eq. 15 are always positive definite, so that the update directions are always downhill. It should also be mentioned that, to ensure decreasing values of the cost function, it is better to consider the updates Eq. 14 and Eq. 15 as directions along wich a line search is performed to find the next iterate.

A second class of methods that avoid calculation of second order derivatives is the class of the so-called quasiNewton methods. Again, Eq. 10 is used to find an update direction along which the next iterate is located using a line search. $(\overline{\bar{H}})_{k}^{-1}$, however, is not explicitely calculated, but is obtained by updating an initial positive definite matrix in every iteration, thereby using first order information. Eventually the updates become close approximations to $(\overline{\overline{\boldsymbol{H}}})_{k}^{-1}$. In this paper we used the BFGS updating formula proposed by Broyden, Fletcher, Goldfarb and Shanno [7] and take the unit matrix as starting point. The advantage of such quasi-newton methods over the Gauss-Newton method is that no linear system must be solved in every iteration as in Eq. 14 and Eq. 15. Only a matrix-vector multiplication is needed. 


\section{NUMERICAL EXAMPLES}

The first reconstructed object is an inhomogeneous cube of side $0.6 \lambda_{b}$, with $\lambda_{b}$ the background wavelength. Permittivities are $1.5 \epsilon_{b}$ for the outer cube and $(2-2 j) \epsilon_{b}$ for the inner cube (Fig. 2(a) and Fig. 2(b)). The antennas are placed on circles with radius $R=2 \lambda_{b}$ around the center of the cube. Two measurement configurations are used. Configuration 1 has 6 meridional circles, evenly distributed on the sphere, each with 12 antennas. In this configuration, two dipoles $\hat{\boldsymbol{u}}_{i_{d}, i_{s}}^{D}$ per source position $\boldsymbol{r}_{i_{s}}^{S}$ are used, the first tangential to the meridional circle and the second perpendicular to the first and tangential to the sphere. Configuration 2 has one circle in the $x y$-plane, one in the $x z$-plane and one in the $y z$-plane, each again with 12 antenna-positions. In this configuration only meridional dipoles are used. All antennas act as both source and receiver. The number of datapoints obtained from the first configuration is 20736 and for the second configuration we have only 1296 datapoints. The inversion domain $\mathcal{D}$ is a cube with side $\lambda_{b}$ and is discretized using $10 \times 10 \times 10$ cubes for both the inverse and the forward problem. The data are generated with the same forward solver as used in the reconstruction. Fig. 3 shows the evolution of the cost function and the reconstruction error $\mathrm{err}_{\epsilon}$ for some inversions using the regularized cost function of section 6.1 with $\alpha=10^{-4} . \mathrm{err}_{\epsilon}$ is defined as

$$
e r r_{\epsilon}=\sqrt{\frac{1}{A B C} \sum_{a=0}^{A-1} \sum_{b=0}^{B-1} \sum_{b=0}^{B-1} \frac{\left|\epsilon_{a, b, c}^{r}-\epsilon_{a, b, c}^{0}\right|^{2}}{\left|\epsilon_{a, b, c}^{0}\right|^{2}}}
$$

where $\epsilon_{a, b, c}^{r}$ and $\epsilon_{a, b, c}^{0}$ are the reconstructed and true permittivity values respectively. It is clear that the best reconstruction is obtained when no noise is present and when the system is most overdetermined. For the reconstruction from the data of configuration 2, wich are far from complete, the reconstruction is less good, although the cost function can be made very small. This is the effect of the non-uniqueness of the problem. For the reconstruction from the noisy data, the iteration was terminated once the cost function reached the noise level. Some reconstruction results are depicted in Fig. 2. From Fig. 3 it can also be seen that the quasi-Newton approach with the BFGS formula results in a much slower convergence than the Gauss-Newton optimization: after 100 iterations the reconstruction error was still 0.106 and the cost function was only reduced to $6 \cdot 10^{-5}$. Here the advantage of not having to solve a linear system in every iteration is completely annihilated by the large number of iterations needed to converge to the desired accuracy. This kind of behaviour was noticed in all our inversions with the BFGS-method. Hence the Gauss-Newton method on the regularized cost function seems better suited for $3 \mathrm{D}$ inversion than the BFGS-method.

The second example is an inversion from measurements obtained with the bistatic free space scattering measurement facility of Institut Fresnel [1]. The object is a polyethylene cube $\left(\epsilon=2.4 \epsilon_{b}\right)$ with side $8 \mathrm{~cm}$. The measurements were performed at a frequency of $2 \mathrm{GHz}$. Ear- lier simulations with an FFT solver showed good agreement with the measurements [8]. For the reconstruction only limited data were available: we used 2064 datapoints to invert for 3375 unknowns. Yet, the results are promising, see Fig. 4.

\section{CONCLUSIONS}

The three dimensional inverse scattering problem was solved using Newton-type optimization algorithms. Two such methods were compared and the Gauss-Newton method is found to be favourable. A multiplicative regularization was applied to the least-squares cost function which resulted in satisfactory reconstructions after only a few iterations.

\section{ACKNOWLEDGMENTS}

Jürgen De Zaeytijd is a Research Assistant of the Fund for Scientific Research - Flanders (F.W.O. - Vlaanderen).

\section{REFERENCES}

[1] J.M. Geffrin, P. Sabouroux and C. Eyraud, "Free space experimental scattering database continuation: experimental set-up and measurement precision", Inverse problems, vol. 21, pp. S117-S130, 2005

[2] A. Franchois and A.G. Tijhuis, "A quasi-Newton reconstruction algorithm for a complex microwave imaging scanner environment", Radio Science, vol. 38, no. 2, pp. 8011,2003

[3] A. Franchois and C. Pichot, "Microwave Imaging - Complex Permittivity Reconstruction with a Levenberg-Marquardt Method", IEEE Transactions on Antennas and Propagation, vol. 45, no. 2, pp. 203215, 1997

[4] A. Kirsch, An Introduction to the Mathematical Theory of Inverse Problems, Springer-Verlag, New York, 1996

[5] P. Zwamborn and P.M. van den Berg, "The ThreeDimensional Weak Form of the Conjugate Gradient FFT Method for Solving Scattering Problems", IEEE Transactions on Microwave Theory and Techniques, vol. 40, no. 9, pp. 1757 - 1766, 1992

[6] A.G. Tijhuis, M.C. van Beurden, A.P.M. Zwamborn, "Iterative Solution of Field Problems with a Varying Physical Parameter", Turk J Elec Engin, vol. 10, no. 2, pp. 163-183, 2002

[7] R. Fletcher, Practical Methods of Optimization, 2nd edition, John Wiley, New York, 1990

[8] P. Lewyllie, A. Franchois, C. Eyraud and J.M. Geffrin, "Testing a 3D BCGS-FFT solver against experimental data", Proceedings of ICEAA'05, pp. 421-424, 2005 


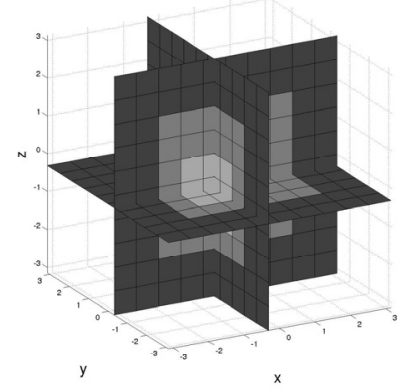

(a) Real part of $\epsilon$-profile

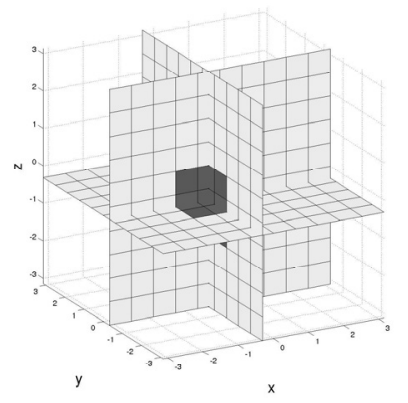

(d) Imaginary part of $\epsilon$-profile
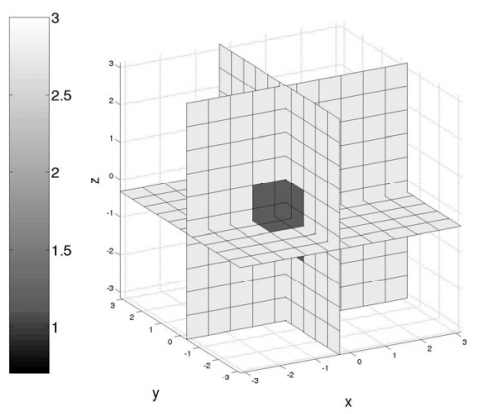

(b) Imaginary part of $\epsilon$-profile

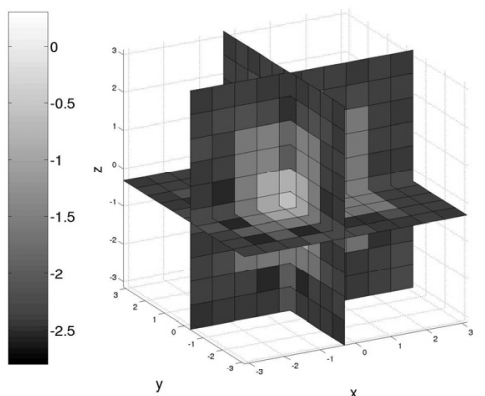

(e) Real part of $\epsilon$-profile

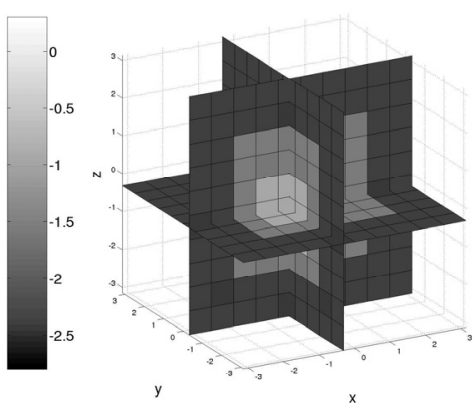

(c) Real part of $\epsilon$-profile
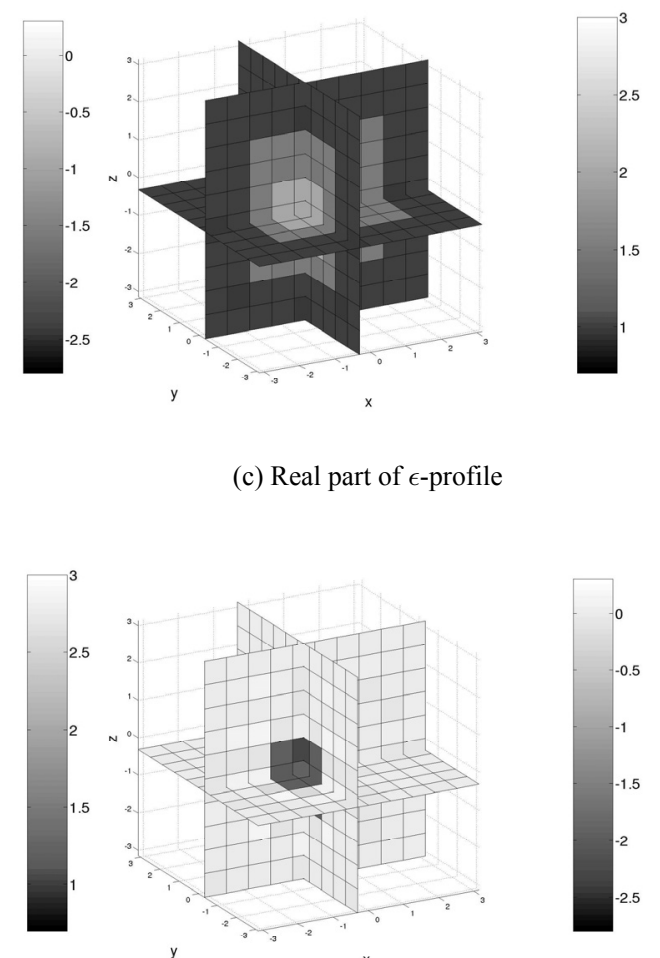

(f) Imaginary part of $\epsilon$-profile

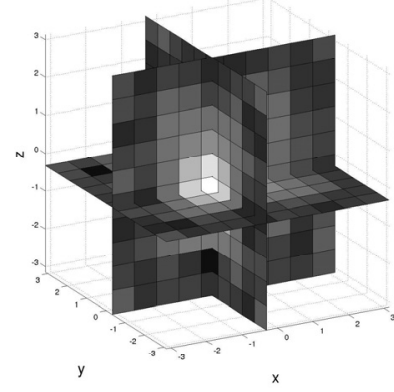

(g) Real part of $\epsilon$-profile

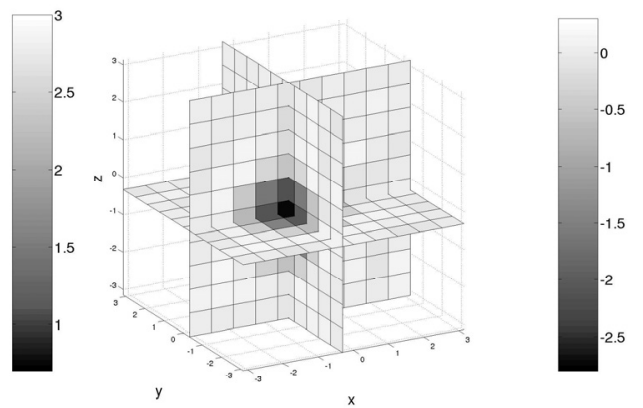

(h) Imaginary part of $\epsilon$-profile

Figure 2. Real (2(a)) and imaginary (2(b)) part of the true (relative) permittivity profile, reconstructed profile after 11 iterations for configuration 1 (heavily overdetermined system) without noise (2(c), 2(d)), reconstructed profile after 13 iterations for configuration 2 (slightly overdetermined stystem) without noise (2(e),2(f)) and reconstructed profile after 3 iterations for configuration 2 with noise: $S / N=30 d B(2(g), 2(h))$. 


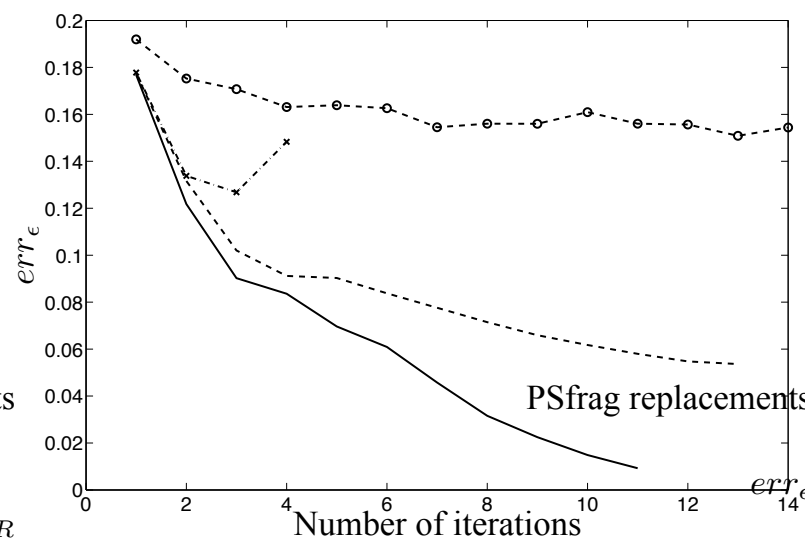

(a) Reconstruction error

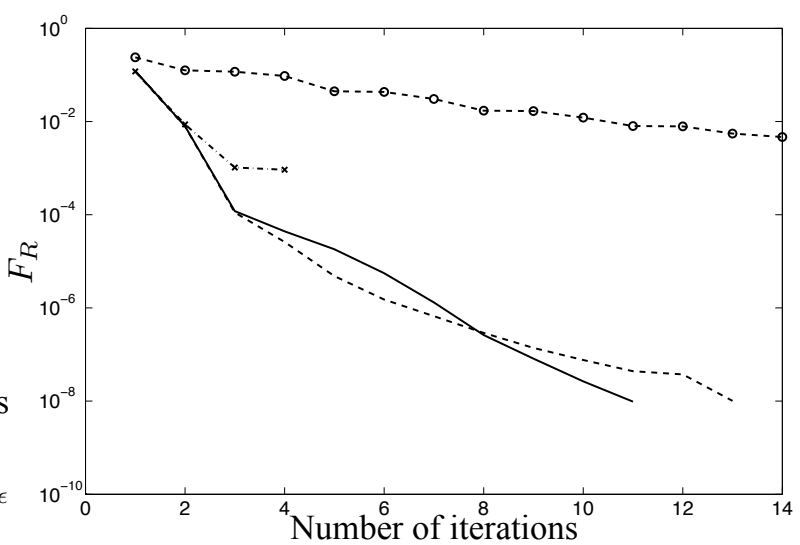

(b) Cost Function

Figure 3. Reconstruction error (3(a)) and cost function (3(b)) for Gauss-Newton in configuration 1 without noise (solid), Gauss-Newton in configuration 2 without noise (dashed), Gauss-Newton in configuration 2 with noise (signal to noise ratio $S / N=30 \mathrm{~dB})(\times)$ and $B F G S$ quasi newton in configuration 2 without noise (o).

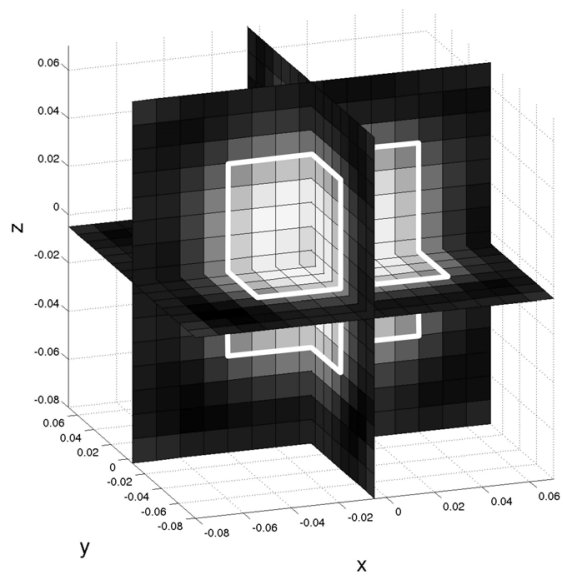

(a) Real part of $\epsilon$-profile
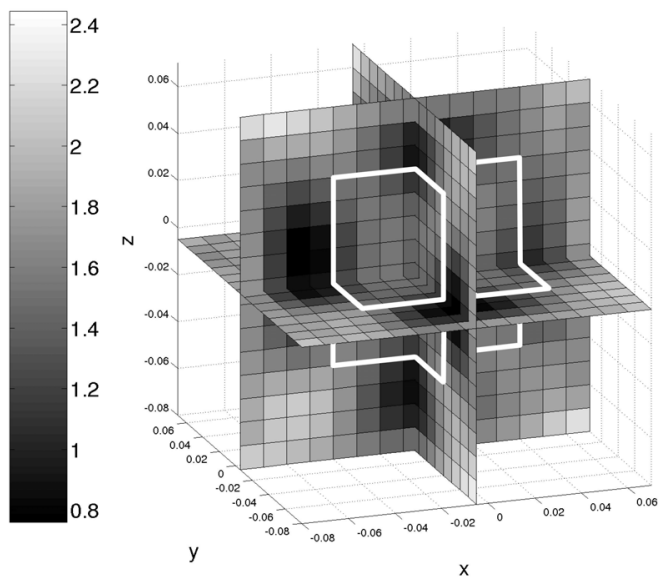

(b) Imaginary part of $\epsilon$-profile

Figure 4. Real (4(a)) and imaginary (4(b)) part of the reconstruced (relative) permittivity profile after 4 iterations from measured data. From comparison of the measurements with simulations the noise level is obtained: $S / N=27 \mathrm{~dB}$. The value of the regularisation parameter was $\alpha=10^{-5}$ 


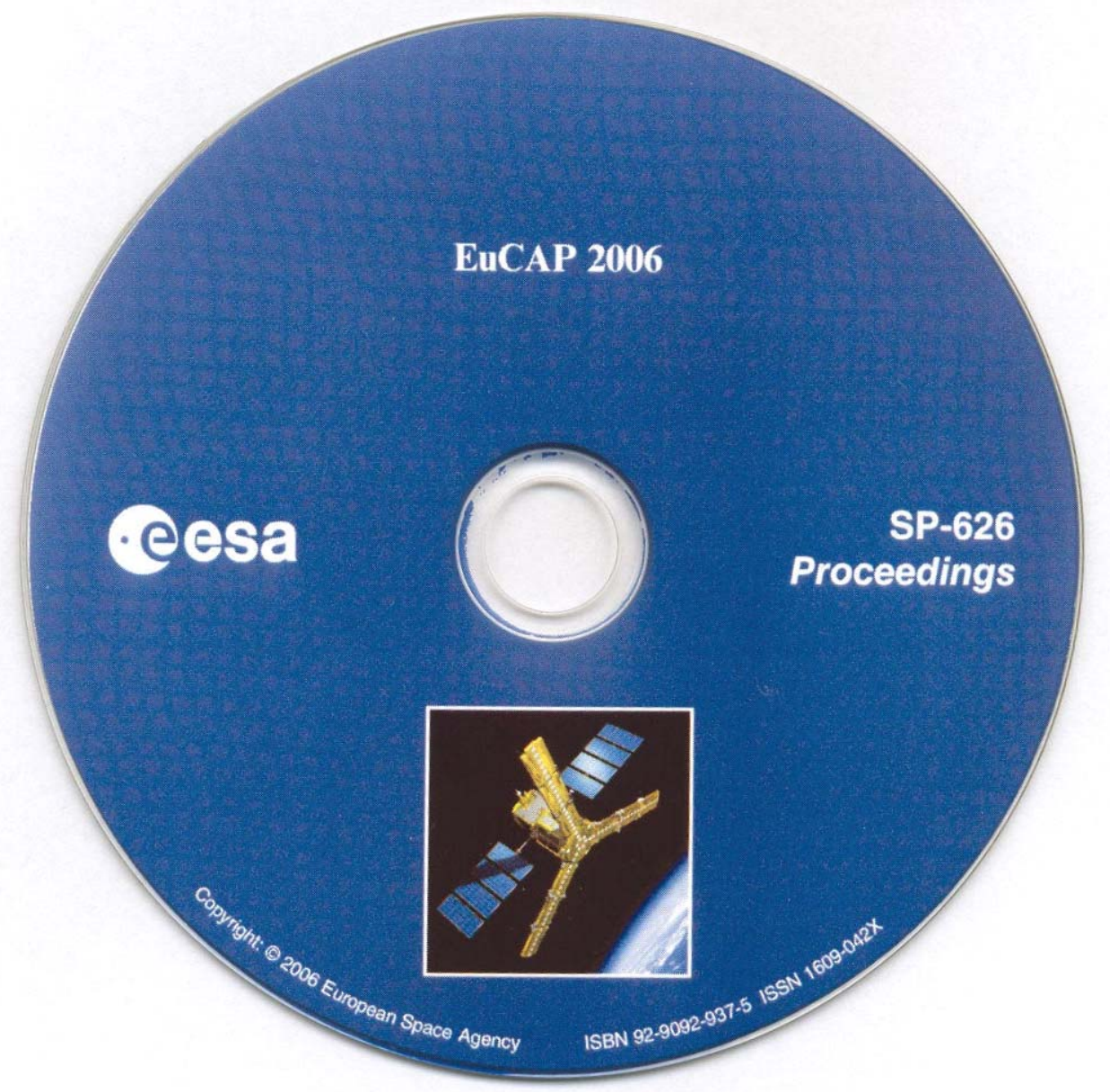




\section{EUCAP CHAIRS}

J. Mosig (General Chair), EPFL, CH

P. Ingvarson (Vice Chair), Saab Ericsson Space, $S$

B. Arbesse r-Rastburg (Vice Chair), ESA-ESTEC, NL

A. Roederer (Honorary Chair), ESA-ESTEC, NL

P. Brachat (Local Chair), France Telecom R\&D, F

C. Pichot (Local Chair), Univ. de Nice, F

Publication

Editors

Published by:

Price:

ISBN No:

ISSN No:

Copyright:
Proceedings of the European Conference on Antennas and Propagation 'EuCap 2006', Nice, France (ESA SP-626, October 2006)

H. Lacoste \& L. Ouwehand

ESA Publications Division

ESTEC

Postbus 299

2200 AG Noordwijk

The Netherlands

The Netherlands

60 Euros

92-9092-9375

1609-042X

(c) 2006 European Space Agency 
SP-626

October 2006

\author{
Proceedings of
}

\title{
The European Conference on Antennas and Propagation: EuCAP 2006
}

\author{
6-10 November 2006 \\ Nice, France
}

European Space Agency Agence spatiale européenne 